\title{
Breaking the Akiyama-Goto cryptosystem
}

\author{
Petar Ivanov and José Felipe Voloch
}

\begin{abstract}
Akiyama and Goto have proposed a cryptosystem based on rational points on curves over function fields (stated in the equivalent form of sections of fibrations on surfaces). It is easy to construct a curve passing through a few given points, but finding the points, given only the curve, is hard. We show how to break their original cryptosystem by using algebraic points instead of rational points and discuss possibilities for changing their original system to create a secure one.
\end{abstract}

\section{Introduction}

A basic ingredient of a public-key cryptosystem is a mathematical procedure that is computationally easy to do but hard to undo. The classical example being the fact that multiplying two integers is easy but factoring an integer is hard. This paper explores the following procedure. Let $R$ be a ring and $a, b \in R$. Then it is easy to find polynomials $X(x, y) \in R[x, y]$ with $X(a, b)=0$. On the other hand, it may be hard, given just $X$, to find the corresponding $a, b$. The case $R=\mathbb{Z}$ of course has been given a lot of attention but, for general $X$ the best known method is not much better than brute-force search. Some improvement can be obtained by sieving and lattice reduction techniques but from the perspective of computational complexity, this does not improve on brute-force search. Much of the discussion of the case $R=\mathbb{Z}$ apply to $R=\mathbb{F}_{p}[t]$ as well. For a discussion of this problem see [4].

A cryptosystem that uses the above problem of "finding points on curves" was proposed by Akiyama and Goto [1] who also proposed a variant in [2]. As we will show in this paper, both variants are insecure. However, the system is not broken by solving the problem of "finding points" and there remains the possibility that a secure cryptosystem can be built around this problem. We briefly discuss this possibility in this paper too. The main motivation of Akiyama and Goto was that, as far it is known, the problem of "finding points on curves" cannot be solved more efficiently by a quantum computer, unlike say, the factoring problem.

2000 Mathematics Subject Classification. Primary 14G50, 94A60; Secondary 11G30.

Key words and phrases. Public-key Cryptosystem, Algebraic Curve, Rational Point.

(c)0000 (copyright holder) 


\section{The cryptosystem of Akiyama and Goto}

In this section we present the cryptosystem described by Akiyama and Goto in $[\mathbf{1}]$. Let $p$ be a prime number, $R=\mathbb{F}_{p}[t]$ be the polynomial ring over the prime field $\mathbb{F}_{p}$ and $K=\mathbb{F}_{p}(t)$ be the field of rational functions over $\mathbb{F}_{p} . K$ is the field of fractions of $R$. Pick a polynomial in two variables $X(x, y) \in R[x, y]$, together with two points $U=\left(u_{x}, u_{y}\right) \in R^{2}$ and $V=\left(v_{x}, v_{y}\right) \in R^{2}$, such that $X(U)=X(V)=0$. In other words, we take an algebraic curve over $K$ together with 2 rational points on the curve. It is easy to find two points and a curve passing through them and we will show how below. On the other hand, if the curve is given (in terms of the polynomial $X(x, y)$ ), it is a hard mathematical problem to find rational points on it. This fact can be used to build the cryptosystem described in this section.

2.1. Keys and key generation. The secret key consists of the two points $U=\left(u_{x}(t), u_{y}(t)\right)$ and $V=\left(v_{x}(t), v_{y}(t)\right)$, such that either $\operatorname{deg} u_{x} \neq \operatorname{deg} v_{x}$ or $\operatorname{deg} u_{y} \neq \operatorname{deg} v_{y}$.

The public key consists of four things: the prime number $p$, the equation $X(x, y)=0$, defining a curve through $U$ and $V$, an integer $l$, which will serve as a lower bound for the degree of a monic irreducible polynomial $f \in R$ and an integer $d$, satisfying

$$
d \geq \max \left\{\operatorname{deg} u_{x}, \operatorname{deg} u_{y}, \operatorname{deg} v_{x}, \operatorname{deg} v_{y}\right\} .
$$

Let us write the equation of the curve as

$$
X(x, y)=\sum_{i, j} c_{i j} x^{i} y^{j}=0
$$

and try to obtain the coefficients $c_{i j} \in R$ in such a way that $U$ and $V$ satisfy it. This means:

$$
\sum_{i, j} c_{i j} u_{x}^{i} u_{y}^{j}=\sum_{i, j} c_{i j} v_{x}^{i} v_{y}^{j}=0 .
$$

If we subtract the second sum from the first we get

$$
\sum_{(i, j) \neq(0,0)} c_{i j}\left(u_{x}^{i} u_{y}^{j}-v_{x}^{i} v_{y}^{j}\right)=0,
$$

which can be written as

$$
c_{10}\left(u_{x}-v_{x}\right)=-\sum_{(i, j) \neq(0,0),(1,0)} c_{i j}\left(u_{x}^{i} u_{y}^{i}-v_{x}^{i} v_{y}^{i}\right) .
$$

Now suppose that $\left(u_{x}-v_{x}\right) \mid\left(u_{y}-v_{y}\right)$. Then the right hand side of (2.3) is also divisible by $u_{x}-v_{x}$, because $u_{x}^{i} u_{y}^{j}-v_{x}^{i} v_{y}^{j}=\left(u_{x}^{i}-v_{x}^{i}\right) u_{y}^{j}+v_{x}^{i}\left(u_{y}^{j}-v_{y}^{j}\right)$. This suggest the following algorithm for choosing $X$ :

(1) For each pair of indices $(i, j) \neq(0,0),(1,0)$ pick a random element $c_{i j} \in R$.

(2) Randomly choose elements $\lambda_{x}, \lambda_{y}, v_{x}, v_{y}$ in $R$, such that $\lambda_{x} \mid \lambda_{y}$.

(3) Compute $u_{y}=\lambda_{y}+v_{y}$ and $u_{x}=\lambda_{x}+v_{x}$.

(4) Compute $c_{10}$ from (2.3).

(5) Compute $c_{00}$ from (2.2) as $-c_{00}=\sum_{(i, j) \neq(0,0)} c_{i j} u_{x}^{i} v_{y}^{j}$. 
2.2. Encryption and decryption. Let $m \in R$ be the secret message that we want to encrypt and assume $\operatorname{deg} m<l$. When $p=2$ we can encode any sequence of $k$ bits as a polynomial of degree $k$ in $\mathbb{F}_{2}[t]$. The assumptions mean that we need to encrypt the secret message by dividing it into blocks of at most $l$ bits and encrypting each of them individually. The encryption goes like this:

(1) Choose a random polynomial $s(x, y) \in R[x, y]$, which satisfies the following condition

$$
\left(\operatorname{deg}_{x} s+\operatorname{deg}_{y} s\right) d+\operatorname{deg}_{t} s<l .
$$

(2) Choose another random polynomial $r(x, y) \in R[x, y]$ and a monic irreducible polynomial $f \in R$, such that

$$
\operatorname{deg}_{t} f>l \text {. }
$$

(3) Compute the cypher polynomial $F(x, y) \in R[x, y]$ according to the formula

$$
F=m+f s+X r .
$$

Now a person knowing the secret key, namely the points $U$ and $V$, can easily decypher the encrypted polynomial $F$ in the following way:

(1) Evaluate $F$ at $U$ and $V$ to get polynomials $h_{1}, h_{2} \in R$.

$$
\begin{aligned}
& h_{1}=F\left(u_{x}, u_{y}\right)=m+f s\left(u_{x}, u_{y}\right) \\
& h_{2}=F\left(v_{x}, v_{y}\right)=m+f s\left(v_{x}, v_{y}\right) .
\end{aligned}
$$

(2) Factor $h_{1}-h_{2}$ and find $f$ as the factor with largest degree.

(3) Compute $m$ as the remainder of $h_{1}$ when divided by $f$.

Note that indeed $f(t)$ is the highest degree factor of $h_{1}(t)-h_{2}(t)$. We have $h_{1}(t)-h_{2}(t)=\left(s\left(u_{x}, u_{y}\right)-s\left(v_{x}, v_{y}\right)\right) f$ and because of the condition (2.5), we only have to show that $\operatorname{deg}\left(s\left(u_{x}, u_{y}\right)-s\left(v_{x}, v_{y}\right)\right) \leq l$. Suppose that the degree of one of the two polynomials, say $s\left(u_{x}, u_{y}\right)$, is greater than $l$. This can only happen if there exist a monomial in $s(x, y) \in R$, say $s_{0}(x, y)=g x^{\alpha} y^{\beta}, g \in R$, such that $\operatorname{deg} s_{0}\left(u_{x}, u_{y}\right)>l$. If this is the case, then use $\alpha \leq \operatorname{deg}_{x} s, \beta \leq \operatorname{deg}_{y} s,(2.1)$ and (2.4) to get:

$l<\operatorname{deg}\left(g u_{x}^{\alpha} u_{y}^{\beta}\right) \leq \operatorname{deg} g+\left(\operatorname{deg} u_{x}\right) \alpha+\left(\operatorname{deg} u_{y}\right) \beta \leq \operatorname{deg}_{t} s+d\left(\operatorname{deg}_{x} s+\operatorname{deg}_{y} s\right)<l$,

which is a contradiction. Thus $f(t)$ is indeed the irreducible factor of $h_{1}(t)-h_{2}(t)$ with highest degree.

The most time consuming part of this decryption algorithm is to factor the polynomial in step 2 . In our case this can be done efficiently using the algorithm of Cantor-Zassenhaus [3]. This is the one of the fastest methods for factoring polynomials over finite fields.

In the description so far we have omitted all the details in choosing the parameters, which concern the security of the cryptosystem and we have listed only the minimal conditions, which have to be imposed to make the decryption possible. However, the algorithm suggested does not always produce a valid decryption. It fails precisely if it happens that $h_{1}-h_{2}=0$, i.e. if $s\left(u_{x}, u_{y}\right)=s\left(v_{x}, v_{y}\right)$. The probability of this happening is negligible with respect to the degree of $s$, as discussed in [1] , where some values for the parameters are suggested. We follow those suggestions for our experiments, described below. 


\section{Breaking the cryptosystem}

We describe an attack which efficiently breaks the protocol just described. However, although it reveals the secret message efficiently, it says nothing about the secret key, as we will see. Finding two or even one rational point on the curve $X(x, y)=0$ is a hard problem, which it turns out one does not need to solve in order to reveal the secret message $m$. The idea is to work in an extension of $R$, in which we can find points on the curve and then use these points to evaluate the cypher polynomial.

Let $S=R[y] /(X(x, 0))$ and let $\alpha=\pi(x)$ be the image of $x$ in $S$ under the natural projection

$$
\pi: R[x] \rightarrow R[x] /(X(x, 0)) .
$$

The point $(\alpha, 0)$ is on the curve $X(x, y)=0$, because by construction $X(\alpha, 0)=$ $\pi(X(x, 0))=0$. We evaluate the cypher polynomial $F$ at $(\alpha, 0)$ to get

$$
F(\alpha, 0)=m+f s(\alpha, 0)+X(\alpha, 0) r(\alpha, 0)=m+f s(\alpha, 0) .
$$

We now want to go back to our original ring by applying the trace operator. To be precise, recall that we denoted $K=\mathbb{F}_{p}(t)$ and let $L=S \otimes_{R} K$. We have the trace operator $\operatorname{Tr}: L \rightarrow K$, which satisfies $\left.\operatorname{Tr}\right|_{K}=[L: K] i d$.

Now choose an element $0 \neq \beta \in S$ with $\operatorname{Tr}(\beta)=0$. If $\gamma \in S$, but $\gamma \notin R$, then $\beta=\gamma-\frac{\operatorname{Tr}(\gamma)}{n}$, where $n=\operatorname{deg}_{x} X(x, 0)$, is such a choice, provided $(p, n)=1$ which we assume for simplicity. Indeed,

$$
\operatorname{Tr}\left(\gamma-\frac{\operatorname{Tr}(\gamma)}{n}\right)=\operatorname{Tr}(\gamma)-\operatorname{Tr}\left(\frac{1}{n}\right) \operatorname{Tr}(\gamma)=\operatorname{Tr}(\gamma)-\operatorname{Tr}(\gamma)=0,
$$

because $\operatorname{Tr}\left(\frac{1}{n}\right)=\frac{1}{n}[L: K]=\frac{1}{n} \operatorname{deg}(X(0, y))=1$.

Now using (3.1) we get

$$
\operatorname{Tr}(\beta F(\alpha, 0))=m \operatorname{Tr}(\beta)+f \operatorname{Tr}(\beta s(\alpha, 0))=f \operatorname{Tr}(\beta s(\alpha, 0)) .
$$

In other words, for any choice of $\beta$, the adversary can compute $p_{\beta}=\operatorname{Tr}(\beta F(\alpha, 0))$, which is a polynomial in $t$ divisible by $f$. But $f$ is monic irreducible polynomial of large degree, which allows the adversary to find it, in case $p_{\beta} \neq 0$. For example he could compute $p_{\beta} \neq 0$ for several different choices of $\beta$, take the greatest common divisor of them, and extract $f$ as the irreducible polynomial of largest degree, which divides the greatest common divisor. The most time consuming computation in this process is the factorization needed to obtain the largest degree irreducible divisor, which is a computation used also in the decryption, as we already saw. We showed how to obtain candidate values for $\beta$ starting with any $\gamma \in S \backslash R$. A simple choice for $\gamma$ is to take the powers of $\alpha$ and in our experiments we never needed to try more than 10 different values for $\gamma$.

Now when $f$ is known to the adversary, he can easily obtain $m$ in the following way. Apply $\operatorname{Tr}$ operator to equation (3.1) to get

$$
\operatorname{Tr}(F(\alpha, 0))=\operatorname{Tr}(m+f s(\alpha, 0))=n m+n f \operatorname{Tr}(s(\alpha, 0)) .
$$

Now $n m$ is the remainder of $F(\alpha, 0)$ when divided by $f$, because of the conditions $\operatorname{deg} m<l<\operatorname{deg} f$.

The computational steps required for the attack are similar to those required for the decryption, except for the computation of traces. The traces of $\alpha^{i}, i=$ $1, \ldots, n-1$ are obtained from the coefficients of $X(x, 0)$ by Newton's identities and, from those values, the trace of any element of $S$ can be easily obtained by 
linearity. We implemented the encryption, decryption and attack steps in Pari/GP (code available at http://www.ma.utexas.edu/users/voloch/GP/asc.gp) In the key generation part of the testing script we use the following choice of parameters (which are in the range suggested by [1]): $w=\operatorname{deg} X$ is a random number between 5 and $8, d$ is chosen to be 50 , the coefficients of $X(x, y) \in R[x, y]$, which are polynomials of $t$ are chosen randomly in such a way that their degree is less than or equal to $d w$. Finally $l$ is chosen to be a random number between $(2 w+4) d$ and $(2 w+4) d+100$. Finally we took for $p$ all primes up to 31 and also 103, 503,997 and 7919 to see how it scaled with $p$. We performed ten trials for each prime. Our experiment suggests that the time it takes to break the system using our attack takes no longer than six times it takes to decrypt the corresponding message using the secret key.

\section{Variants and other attacks}

In addition to the attack described above, the Akiyama-Goto cryptosystem is subject to a different attack due to Uchiyama and Tokunaga, [6], which is not as efficient as ours. Both this attack and ours are discussed in [2], where the authors also discuss a new variant of their cryptosystem immune to those attacks. Briefly, this new variant uses the same shape of encryption $F=m+f s+X r$, except that now $m, f \in \mathbb{F}_{p}[t, x, y]$. To enable decryption, they need to send two encryptions $F_{i}=m+f s_{i}+X r_{i}, i=1,2$. This new cryptosystem is subject to the following attack:

Let $g=f s_{2}-f s_{1}$. We use a substitution attack as in 6.1.1 of [2]. We have $F_{2}-F_{1}=g+X\left(r_{2}-r_{1}\right)$ We begin by substituting points with coordinates in a finite field satisfying the equation $X=0$. These points can be easily found by arbitrarily choosing two of the coordinates and solving for the third. We need as many points as there are coefficients in $g$. As we know $F_{2}-F_{1}$, we can compute its value at these points and therefore we get a set of linear equations for the coefficients of $g$. So we first find $g$ by solving this system and then we use a multivariate polynomial factoring algorithm (as in, for example, [5]) to find $f$ from $g$. Once $f$ is found, then we can find $m$ by plugging points satisfying $X=0$ on $F_{i}=m+f s_{i}+X r_{i}$ which now are linear in $m$ and $s_{i}$. As this attack only determines $g$ up to a multiple of $X$, it will not be efficient if the parameters are chosen so that there too many possibilities for $g$.

Another idea of how make the system secure against the attack described in section 3 is the following: Go back to the original system and make the cyphertext be of the form $m+f s+X r+\left(x^{p^{n}}-x\right) b+\left(y^{p^{n}}-y\right) c$ where $b$ and $c$ are random polynomials in $t, x, y$. If $n$ is sufficiently large, when we plug in the points $U, V$ we can recover the value of $m+f s$ as the remainder of division by $t^{p^{n}}-t$, then proceed as before. This is secure against the attack described in section 3 since the value of $x^{p^{n}}-x$ for $x=\alpha$ as in section 3 will not be divisible by $t^{p^{n}}-t$, ensuring that only rational points can be used for the decryption. On the other hand, $n$ cannot be made too large or the system might be subject to a substitution attack. It is not clear whether this new system is efficient or secure and it merits further study.

\section{References}

1. Akiyama, K., Goto, A.: A Public-key Cryptosystem using Algebraic Surfaces (Extended Abstract), PQCrypto Workshop Record, 2006

2. Akiyama, K., Goto, A.: An improvement of the algebraic surface public-key cryptosystem, Proceedings of SCIS 2008. 
3. Cantor, D., Zassenhaus, H.: A New Algorithm for Factoring Polynomials Over Finite Fields, Mathematics of Computation, 36:587-592, 1981.

4. Elkies, N.D.: Rational points near curves and small nonzero $\left|x^{3}-y^{2}\right|$ via lattice reduction, Algorithmic number theory (Leiden, 2000), LNCS 1838 (2000), 33-63.

5. Gathen, J. v. z., Kaltofen E., Factorization of multivariate polynomials over finite fields, Math. Comp. 45 (1985), no. 171, 251-261.

6. Uchiyama, S., Tokunaga H., On the Security of the Algebraic Surface Public-key Cryptosystems (Japanese), 2C1-2, SCIS2007 (2007).

Dept. of Mathematics, Univ. of Texas, Austin, TX 78712-0257

E-mail address: pivanov@math.utexas.edu

Dept. of Mathematics, Univ. of Texas, Austin, TX 78712-0257

E-mail address: voloch@math.utexas.edu 